\title{
Probing Liquid Surfaces and Interfaces Using Time-of-Flight Secondary Ion Mass Spectrometry
}

\author{
Xiao-Ying $\mathrm{Yu}^{1}$, Zihua $\mathrm{Zhu}^{2}$, Bingwen Liu ${ }^{1}$, Matthew Marshall ${ }^{3}$, Xin Hua ${ }^{1}$, Zhaoying Wang ${ }^{2}, \mathrm{Li} \mathrm{Yang}^{2,6}$ \\ Abigail Tucker ${ }^{3}$, William Chrisler ${ }^{3}$, Eric Hill ${ }^{3}$, Eugene Rodek ${ }^{4}$, Theva Thevuthasan ${ }^{2}$, Yuehe Lin ${ }^{5}$, and \\ James Cowin 6 \\ 1. Atmospheric Sciences and Global Climate Change Division, Pacific Northwest National Laboratory, \\ Richland, WA 99354, USA. \\ 2. W. R \& Wiley Environmental Molecular Science Laboratory, Pacific Northwest National Laboratory, \\ Richland, WA 99354, USA. \\ 3. Biological Sciences Division, Pacific Northwest National Laboratory, Richland, WA 99352, USA. \\ 4. SPI Supplies/Structure Probe, Inc., West Chester, PA 19380, USA. \\ 5. School of Mechanical and Materials Engineering, Washington State University, WA, 99164, USA. \\ 6. Chemical and Materials Sciences Division, Pacific Northwest National Laboratory, Richland, WA \\ 99354, USA.
}

The surfaces of aqueous phases and films can have unique kinetics and thermodynamics, distinct from the bulk. However, major surface analytical techniques are mostly vacuum-based and direct applications for volatile liquid studies are difficult. We developed a vacuum compatible microfluidic interface to enable surface analysis of liquids and liquid-solid interactions. The unique aspect of our approach is that 1) the detection window is an aperture of $2-3 \mu \mathrm{m}$ in diameter, which allows direct detection of the liquid surface, and 2) it uses surface tension to hold the liquid within the aperture. The microfluidic reactor is composed of a silicon nitride ( $\mathrm{SiN})$ membrane and polydimethylsiloxane (PDMS) [1, 2]. Its application in ToF-SIMS as an analytical tool was evaluated. Most recently, we demonstrated in situ probing of the electrode-electrolyte solution interface using a new electrochemical probe based on our original invention. It provides direct observation of the surface and diffused layer with chemical speciation in liquids using ToF-SIMS for the first time. Moreover, we extended the microfluidic reactor for biofilm growth and real-time characterization. Our results provided the first ToF-SIMS molecular imaging of the hydrated biofilm using this unique capability.

A ToF-SIMS V spectrometer (IONTOF GmbH, Münster, Germany) was used in these experiments. A pulsed $25 \mathrm{keV} \mathrm{Bi}+$ (beam size: $\sim 250 \mathrm{~nm}$ ) ion beam with an incident angle of 45 degree off the normal was used as the primary ion beam for all measurements. The SIMS measurements were performed at the beam current of $\sim 1.0 \mathrm{pA}$ with a beam width of $130 \mathrm{~ns}$ and a repeated frequency of $20 \mathrm{kHz}$. The main chamber vacuum pressure was $2-4 \times 10^{-7}$ mbar with our device inside the chamber, and the pressure only slightly increased to $3-5 \times 10^{-}$mbar during measurements. This indicates that no spraying or fast spreading of aqueous solutions occurs through the aperture.

Figure 1 depicts the latest results from the first electrode-electrolyte solution interface observation using in situ ToF-SIMS. The electrolyte is a dilute KI solution. The three electrode electrochemical system consists of a gold $(\mathrm{Au})$ working electrode, a platinum $(\mathrm{Pt})$ counter electrode and a $\mathrm{Pt}$ reference electrode. Figure 2 depicts the first in situ hydrated biofilm SIMS m/z spectrum and 2D images of selected fatty acids (FA) fragments, which confirms the molecular imaging of biofilm in addition to in situ confocal scanning laser spectroscopy observations of biofilm growth. These new results demonstrated technical 
advancements of conducting chemical imaging of liquid-solid interface in situ in their native liquid environment using surface sensitive characterization tools [3].

\section{References:}

[1] L Yang et al., J. Vac. Sci. Technol. A 29 (2011), art. no., 061101, doi: 10.1116/1.3654147.

[2] L Yang et al., Lab Chip 11 (2011), p. 2481. doi: 10.1039/c01c00676a.

[3] B Liu et al., Lab Chip (2014), 14(5), 854-859. doi: 10.1039/C3LC50971K.

[4] H Xin et al., Analyst (2014), advanced on-line publication. doi: 10.1039/C3AN02262E.

[5] The authors acknowledge funding from the Pacific Northwest National Laboratory (PNNL)

Technology Development Program (TIP) fund and the Chemical Imaging Initiative LDRD fund.
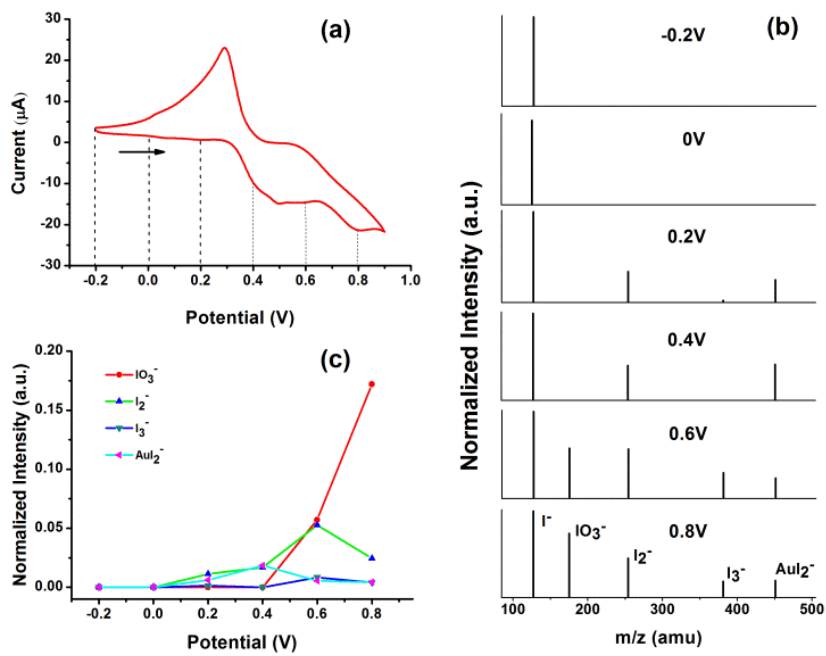

Figure 1. (a) The cyclic voltammogram for the polycrystalline Au electrode in a $10 \mathrm{mM} \mathrm{KI}$ electrolyte solution. (b) ToF-SIMS $\mathrm{m} / \mathrm{z}$ spectra acquired at different potentials. Only $\mathrm{m} / \mathrm{z}$ peaks of interest are shown. (c) Normalized intensity from the $\mathrm{m} / \mathrm{z}$ spectra obtained in ToF-SIMS as a function of potential. The I signal was used as a reference for normalization of each spectrum [3].

(a)

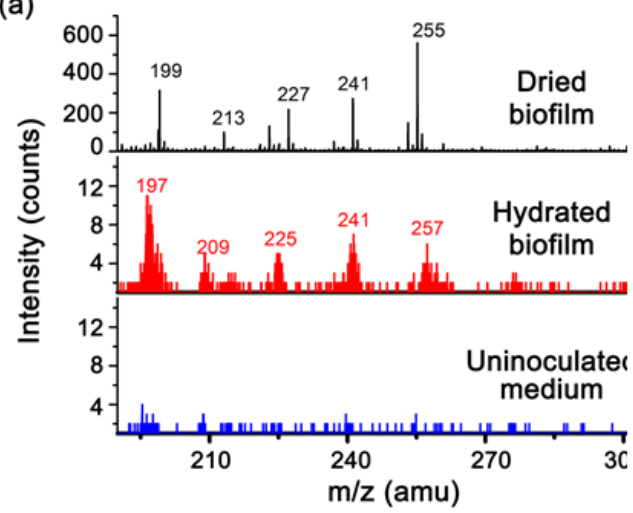

(b)

$\mathrm{m} / \mathrm{z} 225$
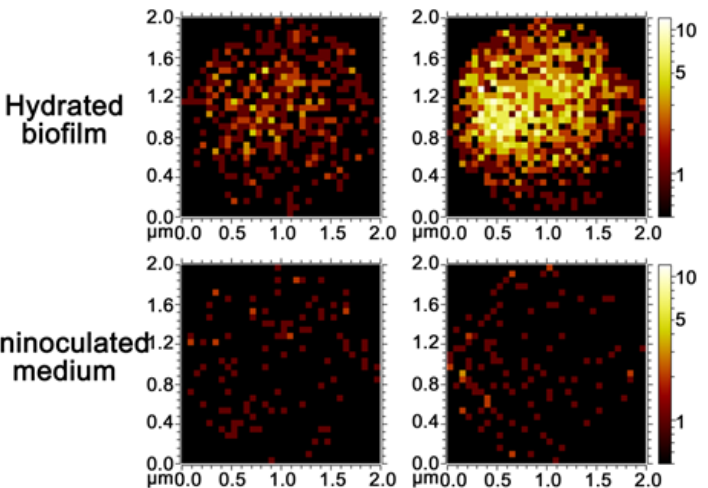

Figure 2. (a) Representative ToF-SIMS $\mathrm{m} / \mathrm{z}$ spectra of dried Shewanella sp. biofilm deposited on clean $\mathrm{Si}$ wafer, hydrated biofilm, and uninoculated medium solution and (b) $2 \mathrm{D}$ images of $\mathrm{m} / z 225$ (C $\mathrm{C}_{14} \mathrm{FA}_{\text {) }}$ 